VALENZUELA, Jonatan. "Hacia un estándar de prueba cautelar en materia penal: algunos apuntes para el caso de la prisión preventiva".

Polit. crim. Vol. 13, No 26 (Diciembre 2018) Art. 5, pp. 836-857.

[http://www.politicacriminal.cl/Vol_13/n_26/Vol13N26A5.pdf]

\title{
Hacia un estándar de prueba cautelar en materia penal: algunos apuntes para el caso de la prisión preventiva
}

\section{Towards a standard of precautionary trial in criminal matters: some notes for the case of preventive detention}

\author{
Jonatan Valenzuela Saldias \\ Universidad de Chile \\ jvalenzuela@derecho.uchile.cl
}

\section{Resumen}

En este trabajo se explora la estructura básica de una regla de estándar de prueba en materia cautelar penal. Para ello debemos enfrentar la posibilidad de afirmar la pertinencia de hablar de "prueba" en una etapa anterior al juicio oral y proponer un ejercicio de definición de los criterios de aplicación de la medida cautelar personal de prisión preventiva.

Palabras clave: Proceso penal - prueba - medidas cautelares - prisión preventiva

\begin{abstract}
This paper describes the basic structure of a rule standard of proof in criminal precautionary material is explored. To do this we must face the possibility of affirming the relevance of talking about "proof" in a pre-trial stage and propose an exercise in defining the criteria for applying personal precautionary measure of preventive detention.
\end{abstract}

Keywords: Criminal Process - evidence - protective orders - preventive detention

\section{Introducción}

Las medidas cautelares personales se han ido convirtiendo en un tema central del derecho procesal penal. La resonancia pública que tiene la decisión de aplicación de una medida que supone encerrar a una persona tiene un atractivo que obliga a explicar a la sociedad muchas de las instituciones centrales del proceso. Lo más llamativo es contrastar esas explicaciones con las intuiciones compartidas de la comunidad respecto del uso del ius puniendi estatal. ${ }^{1}$

Por otro lado, el razonamiento probatorio ha sido corrientemente estudiado en el ámbito de la justificación de las sentencias o decisiones finales en el proceso, tanto civil como penal. Es común estudiar a las pruebas y a la actividad probatoria pensando en la justificación de

\footnotetext{
${ }^{1} \mathrm{Y}$ ha despertado interés desde las diversas "ciencias criminales", véase al respecto KOSTENWEIN, Ezequial, "Prisión preventiva: entre los medios de comunicación y las autoridades políticas" Direito e Práx, Vol. 08, N. 2 (2017) pp. 942-973
} 
VALENZUELA, Jonatan. "Hacia un estándar de prueba cautelar en materia penal: algunos apuntes para el caso de la prisión preventiva".

la sentencia definitiva, aunque es también un tema de relevancia cuando se trata de enfrentar a la decisión judicial de imposición de una medida cautelar. ${ }^{2}$

El denominado cognoscitivismo crítico se ha ocupado intensamente de desentrañar el sentido que del enunciado "está probado que p", como base del uso del grupo de reglas probatorias que debe ser enfrentado de cara a la justificación de las decisiones probatorias en cierto sentido terminales del procedimiento. ${ }^{3}$

La frase está probado que p, en la mayoría de las ocasiones, denota el uso de un tipo de razonamiento que se asocia al momento de enfrentar la corrección de la proposición fáctica que integra una sentencia. Es el clásico razonamiento sobre hechos cuyo conocimiento es entendido como presupuesto de la justificación de decisiones. ${ }^{4}$

Sin embargo, este no es el único tipo de enunciado probatorio que un juez debe enfrentar. Desde una perspectiva general, toda decisión basada en la aplicación de normas abstractas se enfrenta a la necesidad de reconocer un razonamiento basado en evidencias que permita controlar las razones para adoptar la decisión. Esto ocurre, por cierto, en todas las actividades humanas en las que busquemos conocer la racionalidad de la misma decisión.

En la doctrina chilena el tema ha sido advertido. Así es como se ha dicho que "hablar de los estándares de convicción es hablar de la prueba de los hechos" y con ello se nos remite a la necesidad de contar con criterios de control intersubjetivo cuando se trata de las medidas cautelares. En este sentido, esa vinculación entre "convicción" y prueba de los hechos da cuenta de la necesidad de reconocer un cierto control probatorio de las premisas de hecho que concurren en todas las decisiones procesales. ${ }^{5}$

Dentro de las medidas cautelares personales del sistema procesal penal sin duda la que ofrece mayor interés es la prisión preventiva. Esto, a mi juicio, por dos razones: en primer lugar se suele sostener que la prisión preventiva es la medida cautelar personal que afecta de modo más intenso los derechos fundamentales del imputado al habilitar al estado a encerrar a una persona en la cárcel. Incluso se ha llegado a sostener que se trata de una "anticipación punitiva" por el fuerte parecido que tiene con la pena de prisión. Este parecido es claramente fenomenológico, es decir se basa en lo que puede describirse como la manera en que tiene lugar el ejercicio de la prisión preventiva y la manera en que tiene

\footnotetext{
${ }^{2}$ En este sentido, véase BELTRÁN, Ramón, "Estándares de prueba y su aplicación sobre el elemento material de la prisión preventiva en Chile", Política Criminal, Vol. 7, No 14 (2012) pp. 454 - 479, quien refiriéndose a una parte del razonamiento propio de la medida cautelar da cuenta del razonamiento que justifica su imposición.

${ }^{3}$ Por todos véase Ferrer, Jordi, Prueba y verdad en el Derecho, Barcelona: Marcial Pons, 2005, pp. 23 - 40.

${ }^{4}$ Esta es una visión tradicional acerca de la necesidad de reconocer la pretensión de relevancia de la verdad en el proceso. Véase en este sentido TARUFFO, Michele, Simplemente la verdad, Barcelona: Marcial Pons, 2010 , pp. 89 - 153. Llamativamente nuestra doctrina procesal penal no es conteste en este punto, centrando el sentido del proceso penal en el "conflicto", por todos véase MATURANA, Cristián y MONTERO, Raúl, Derecho Procesal Penal. Tomo I, Santiago: Abeledo Perrot, 2010, pp. 1 - 5.

${ }^{5}$ Véase BELTRÁN, R., "Estándares de prueba y su aplicación sobre el elemento material de la prisión preventiva en Chile", en Política Criminal, Vol. 7, Nº 14 (2012) pp. 454 - 479.
} 
Polit. crim. Vol. 13, No 26 (Diciembre 2018) Art. 5, pp. 836-857.

[http://www.politicacriminal.cl/Vol_13/n_26/Vol13N26A5.pdf]

lugar la pena. Lo que se juega el imputado cuando se lo sujeta a esta medida cautelar es de alta importancia, tal como ocurre en el momento de adjudicación de la pena. ${ }^{6}$

En segundo lugar, la prisión preventiva tiene importancia frente a la determinación del exacto sentido de la presunción de inocencia como regla de trato. Ello hace que el razonamiento probatorio del juez que debe decidir sobre la cautelar tenga una estructura diferente a la que se enfrenta en la etapa de juicio para imponer una pena, debido a que la proyección de la presunción de inocencia en la etapa de juicio traspasa a la mera regla de trato y se convierte en una regla, precisamente, de juicio. ${ }^{7}$

Lo anterior puede explicarse asumiendo la necesidad de justificar decisiones que sean compatibles con la afirmación de inocencia del acusado y con el hecho de padecer una medida de prisión preventiva. En el caso de la pena, esta relación no es posible pues para proceder a la imposición de la pena debe declararse la culpabilidad del acusado. ${ }^{8}$

En el contexto de la prisión preventiva, la inocencia del sujeto se mantiene inalterada debido a que no existe sentencia que lo señale como culpable. Dicho en otros términos, el filtro que impone la presunción de inocencia como regla de juicio no existe dentro del abanico de consideraciones del juez a la hora de decidir una medida cautelar de prisión preventiva. Esto no quita que, por otro lado, pueda tematizarse la relevancia de la medida cautelar desde el punto de vista de la regla de trato que se deriva de la presunción de inocencia. En este sentido puede advertirse que en términos conceptuales el trato que se dispensa al imputado al decretarse una prisión preventiva coexiste con su estatuto de inocente en tanto no pueda advertirse un juicio sobre la culpabilidad del mismo radicado en la sentencia. ${ }^{9}$

\footnotetext{
${ }^{6}$ Subsecuentemente el error que puede cometer el juez al imponer una medida de prisión preventiva que no debía ser impuesta (falso positivo) o al no imponer una que debía ser impuesta (falso negativo) pareciera compartir el tipo de error de la sentencia. Creo, de todos modos, que existe una diferencia esencial pues en la prisión preventiva no se cuestiona el estatuto de inocente del imputado como sí se hace en la pena. Me he referido a esta relación en otro trabajo, véase VALENZUELA, Jonatan, "Presumir responsabilidad: sobre una relación entre la prisión preventiva y la presunción de inocencia en el derecho procesal chileno", en Revista de Estudios de la Justicia, núm. 14 (2011) pp. 53 - 70.

${ }^{7}$ También me he referido a este punto en el proceso chileno. Véase VALENZUELA, Jonatan, "Inocencia y razonamiento probatorio" en Revista de Estudios de la Justicia, número 18 (2013) pp. 13 - 23.

${ }^{8}$ Este problema se proyecta en el ámbito de las medidas de seguridad en el mismo sistema procesal penal. Sobre el particular véase VALENZUELA, Jonatan, "Hechos, pena y proceso", Santiago: Rubicón, 2017, pp. $111-123$.

${ }^{9}$ Sobre este punto resulta particularmente claro DEI VECCHI, Diego, “Acerca de la justificación de la prisión preventiva y algunas críticas frecuentes” en Revista de Derecho (Valdivia), Vol. XXVI, N² 2 (2013) pp. 189 217, quien sostiene: "Los fundamentos en que estas afirmaciones se basan se reducen en general a las idénticas consecuencias que el encarcelamiento tiene para la persona encerrada, sea cual fuere el fin institucional en cada caso46. Sin embargo, estas críticas no solo no logran dar en el blanco sino que además oscurecen notablemente el punto de discusión. En primer lugar, la crítica no da en el blanco porque arguye la falta de justificación de la prisión preventiva negando una distinción conceptual que, al menos en principio, puede asumirse como meramente descriptivas...” p. 202.
} 
VALENZUELA, Jonatan. "Hacia un estándar de prueba cautelar en materia penal: algunos apuntes para el caso de la prisión preventiva".

Desde el punto de vista del razonamiento judicial se suelen dirigir los esfuerzos a los denominados "supuestos materiales" de las mismas medidas, en concreto de la prisión preventiva. $^{10}$

Así las cosas, existen enunciados sobre hechos, en diversos escenarios procesales. En la decisión propia de las denominadas "medidas cautelares", se habla de antecedentes, bases de presunciones, datos entre otros, como base de los criterios que permiten conceder o no una determinada medida cautelar. Luego, con mayor o menor énfasis, estas palabras designan maneras de corroborar enunciados sobre hechos que deben haber acaecido para justificar el uso de la medida cautelar. ${ }^{11}$

Junto con lo anterior, cabe señalar que las decisiones cautelares son provisionales. Esto es, dependen de las condiciones bajo las cuales resultan procedentes y si esas condiciones cambian la decisión puede volverse injustificada. La existencia de esas condiciones, su mantenimiento o su fin suponen un pronunciamiento racional del juez y en muchas ocasiones ese pronunciamiento requiere la corroboración de una premisa sobre hechos. Debe entenderse que los enunciados sobre hechos que sirven de base para la aplicación de las medidas cautelares en el proceso deben ser capaces de soportar un juicio basado en pruebas.

\section{Error y medida cautelar}

Como toda decisión procesal, la imposición de una medida cautelar como la prisión preventiva se relaciona con la producción de errores. La decisión cautelar tiene una doble dimensión en lo relativo al enfrentamiento de errores del proceso. Por una parte, la decisión sobre una cautelar debería servir para aumentar las probabilidades de acierto de la decisión de la sentencia. Esto quiere decir, que si se adjudica una medida de prisión preventiva, por ejemplo, debido al riesgo de manipulación o destrucción de evidencias resulta necesario establecer una relación entre la concesión de esa medida cautelar y las probabilidades de aumento de acierto, en términos de evitación del error judicial, de la sentencia. La concesión de la prisión preventiva es instrumental en este preciso sentido: cautela al procedimiento en términos de una regla de evitación de errores, debiendo mejorar la información de la sentencia en el juicio, aumentando sus probabilidades de acierto.

Por otro lado, si asumimos que la decisión cautelar, en sí misma, se sujeta al razonamiento probatorio debe contar con criterios que permitan distribuir errores desde el punto de vista cautelar. Esto quiere decir que la concesión o denegación de una medida cautelar puede ser vista como una decisión que además tiende a la distribución de errores desde el punto de vista cautelar, esto es, que debe existir un marco de suficiencia para decidir la concesión o denegación de una medida cautelar que suponga distribuir los casos en que existirá falso

\footnotetext{
${ }^{10}$ No siempre aparece clara la distinción, por ejemplo, es el caso de MATURANA, Cristian y MONTERO, Raúl, "Derecho procesal penal, Tomo I", Santiago: Abeledo Perrot, 2010, pp. 481 - 482; 485 - 499.

${ }^{11}$ En este sentido, las nociones de "peligro en la demora" o de "humo del buen derecho" a modo de ejemplo, tienen una clara vocación empírica en el razonamiento judicial. Véase sobre estos conceptos MARIN, Juan Carlos "Las medidas cautelares en el proceso civil chileno", Santiago, Editorial Jurídica de Chile, 2005, pp. 145 - 182; ARAZI, Roland "Medidas Cautelares", Buenos Aires, Astrea, 2014, p.5.
} 
Polit. crim. Vol. 13, No 26 (Diciembre 2018) Art. 5, pp. 836-857.

[http://www.politicacriminal.cl/Vol_13/n_26/Vol13N26A5.pdf]

positivo (una medida cautelar concedida que no debía concederse) o un falso negativo (denegación de una medida cautelar que debía concederse).

En el entendido anterior, la medida cautelar es capaz de producir una determinada clase de error que podemos llamar "error cautelar", el juez tiene capacidad de generar errores en el escenario cautelar tal como pueden generarse errores en el escenario de la sentencia definitiva.

La pregunta que surge es: ¿con qué criterios probatorios el juez decide la aplicación en un caso determinado de una medida cautelar como la prisión preventiva?

Como he apuntado, la prueba jurídica que, en abstracto, no difiere como proceso cognitivo con la actividad probatoria en cualquier campo del conocimiento, ha estado centrada tradicionalmente en la definición de los marcos en que debe entenderse justificada la proposición "está probado que $p$ " de cara a justificar decisiones fiables en el contexto de la decisión en algún sentido "final" del procedimiento. Suele hablarse de justificación racional de una decisión probatoria en una sentencia. Sin embargo, nada impide que podamos utilizar ese modelo de razonamiento en el ámbito de las decisiones cautelares.

Como bien ha apuntado Laudan: "Hasta ahora, nos hemos centrado en errores que pueden tener lugar en la fase o etapa terminal, es decir, nos hemos venido preocupando por los veredictos erróneos. Sin embargo, muchas investigaciones penales nunca llegan tan lejos. En ocasiones, las pesquisas de la policía van perdiendo fuerza hasta estancarse debido a la falta de mayores indicios y pistas, o debido también al seguimiento de prácticas de investigación deficientes. Aunque estas situaciones constituyen también errores, tal y como una absolución falsa lo es, no estarán en el centro de este análisis." ${ }^{2}$

Esta tendencia ha dejado de lado varios escenarios donde la racionalidad de las decisiones judiciales debe ser estudiada. Es el caso de las medidas cautelares, las que, además, guardan directa relación con la capacidad de reducir errores de cara al proceso penal en su etapa final.

En este trabajo quisiera dirigir mi atención a la pregunta sobre la existencia de la actividad probatoria en el ámbito de las medidas cautelares y correlativamente a la pregunta sobre la formulación del estándar de prueba en ese contexto.

Esto supone entrar en las condiciones de adjudicación de una medida cautelar de manera interna, es decir, dejando por un momento de lado su capacidad reductora de error de cara al momento final del procedimiento. Resulta necesario, a mi juicio, realizar el ejercicio de manera de poder mostrar qué argumentos parecen estar disponibles para el caso de la justificación racional de decisión sobre medidas cautelares.

${ }^{12}$ Véase LAUDAN, Larry, "Verdad, error y proceso penal”, Barcelona, Marcial Pons, 2013, p. 40. 
VALENZUELA, Jonatan. "Hacia un estándar de prueba cautelar en materia penal: algunos apuntes para el caso de la prisión preventiva".

No me encargaré de la fenomenología de las medidas cautelares, sino que me referiré en abstracto a la base del modelo de razonamiento probatorio que puede exigirse de este tipo de decisiones judiciales pensando en el concreto ámbito de la prisión preventiva.

No me referiré tampoco al problema del contenido de la medida cautelar en términos de desentrañar su parentesco con otras instituciones (como la pena estatal o la tutela anticipada), sino que sólo quiero mostrar el camino para formular un estándar de prueba en materia cautelar.

Por cierto, me parece que la mejor manera de partir esta clase de estudios es centrando nuestra atención en las medidas cautelares personales. En materia penal y en particular en el ámbito de las medidas cautelares encontramos muchos argumentos que permiten cuestionar la manera tradicional de estudio del razonamiento del juez en este ámbito y creo que una visión general puede resultar aplicable con matices a variados contextos normativos.

\section{Actividad probatoria y prisión preventiva}

¿Existe stricto sensu actividad probatoria en el ámbito de la decisión judicial sobre concesión o denegación de una medida cautelar como la prisión preventiva? Creo, por las razones que expondré a continuación que la respuesta a esa pregunta es positiva y lleva, necesariamente, a preguntas subsecuentes: ¿cómo ha de valorarse esa prueba?, ¿cuál es el estándar de prueba más adecuado para ser utilizado por los jueces en esta materia?

El problema se vuelve interesante cuando uno se detiene por un momento en la información que rodea a una medida cautelar, ¿cuál es el modelo de razonamiento que nos permita reconstruir racionalmente las decisiones en éste ámbito cuando se trata de decisiones que se toman al inicio del procedimiento, con, muchas veces, baja actividad probatoria?

El primer rasgo corrientemente apuntado por la doctrina supone que las medidas cautelares tienen un carácter provisional. Esto quiere decir, en la mayoría de los casos que se trata de decisiones judiciales desprovistas de una pretensión de persistencia en el tiempo. Las medidas cautelares subsisten mientras las razones que les justifican permanezcan en el tiempo. $^{13}$

Por otro lado, la decisión judicial en el ámbito de las medidas cautelares suele ser descrita como instrumental. Esto quiere decir que se trata de medidas que sirven a un objeto que no se relaciona directamente con la decisión de conceder o no la medida. En general podemos sostener que se trata de cautelar el sentido del proceso. ${ }^{14}$

En tercer lugar, la decisión judicial sobre una medida cautelar no es exactamente retrospectiva pues obliga al juez al despliegue de cierta actividad prospectiva, dedicada a la

\footnotetext{
${ }^{13}$ Véase por todos MARÍN, Juan, Las medidas cautelares... Cit. Nota 2, pp. 193 - 195.

${ }^{14}$ Ver MARIN, Juan, Las medidas cautelares... pp. 198 - 200. En particular, sobre el proceso penal véase HORVITZ, María y LÓPEZ, Julián, "Derecho Procesal Penal Chileno", Santiago: Editorial Jurídica de Chile, 2002, p. $342-349$.
} 
Polit. crim. Vol. 13, No 26 (Diciembre 2018) Art. 5, pp. 836-857.

[http://www.politicacriminal.cl/Vol_13/n_26/Vol13N26A5.pdf]

evitación de eventos futuros (los que tienen por propiedad principal que pueden ocurrir o no, y para ello la medida cautelar parece tener importancia).

La prisión preventiva suele justificarse en los sistemas occidentales bajo el supuesto que de concurrir indicios o datos que permitan considerar plausible que existe un peligro de fuga del imputado debemos aplicar una medida cautelar de prisión preventiva pues, de otro modo, no tendría sentido desarrollar un procedimiento que puede terminar con una sentencia condenatoria respecto de un ausente. ${ }^{15}$

El peligro supone definir un estado de cosas que permite considerar que resulta razonable evitar el acaecimiento de un hecho que de ocurrir, vulneraría el sentido del proceso. Pensemos en el ejemplo del peligro de fuga. La decisión judicial acerca del peligro de fuga de un imputado en un procedimiento penal supone el despliegue de un argumento que, en alguna medida, debe incluir la aceptación como probable de un hecho en el futuro: la fuga.

El razonamiento probatorio de la sentencia tiene, por regla general, una vocación retrospectiva. La verdad perseguida supone tener por verdaderos enunciados que dicen relación con hechos que esencialmente han tenido lugar en el pasado. En materia penal se trata de dar por establecido si la hipótesis de hecho de la acusación está suficientemente corroborada para declarar culpable al acusado. Lo que se afirma es la verdad de esos enunciados de hecho que han tenido lugar en el pasado. Este punto queda fuertemente comprometido en el caso de la decisión acerca de las medidas cautelares. En muchos casos las medidas cautelares requieren afirmar la verdad (en términos probatorios) de "hechos" que tendrán lugar en el futuro.

El juez debe enfrentar un razonamiento predictivo basado en evidencias. Esto quiere decir que en términos estrictos no parece razonable abandonar la pretensión de racionalidad de toda decisión judicial en el ámbito de las medidas cautelares por el desafío que se plantea a propósito de la existencia de identificar con datos del presente el acaecimiento de hechos futuros. $^{16}$

Esto obliga a entender a la decisión judicial en materia cautelar como una clase de decisión que, necesariamente, se ampara en evidencias. Así, pueden establecerse criterios objetivos de evitación y distribución del error en materia de esta clase de decisiones. Esta perspectiva, por sobre todo, puede proveernos de criterios de evaluación para el uso de las medidas cautelares de manera tal de verificar su corrección. De esta manera podemos reducir el campo que la falta de control sobre los enunciados de hechos abre a la irracionalidad en el terreno procesal.

\footnotetext{
${ }^{15}$ Así por ejemplo, el artículo 140 letra c) del Código Procesal Penal chileno: "Que existen antecedentes calificados que permitieren al tribunal considerar que la prisión preventiva es indispensable para el éxito de diligencias precisas y determinadas de la investigación, o que la libertad del imputado es peligrosa para la seguridad de la sociedad o del ofendido, o que existe peligro de que el imputado se dé a la fuga, conforme a las disposiciones de los incisos siguientes".

${ }^{16}$ En cierta doctrina aparece esta idea detrás de la lógica cautelar. De manera crítica ALVARADO, Adolfo, "Cautela Procesal", Rosario: Juris, 2008, pp. 10 - 16.
} 
VALENZUELA, Jonatan. "Hacia un estándar de prueba cautelar en materia penal: algunos apuntes para el caso de la prisión preventiva".

\section{La racionalidad de las medidas cautelares penales.}

El contexto en el que se encuentra la decisión sobre una determinada medida cautelar está determinado por las exigencias de racionalidad que comparten todas las decisiones en el proceso. La exigencia de un "justo y racional procedimiento" abarca a todo momento procesal y no tan sólo a la etapa eventual de un juicio oral. Esto porque allí donde se adopten decisiones de cara a los hechos pareciera necesario encontrar un espacio para el razonamiento con las evidencias del caso. Se trata de enfrentar la realización de hechos que pueden justificar la imposición de la cautelar y con ello resulta necesario que el juez se haga cargo de todas las afirmaciones sobre hechos que sean pertinentes al caso. ${ }^{17}$

Sin embargo, suele darse el caso en que el legislador usa términos vagos para referirse a los apoyos que las hipótesis de hecho tienen en el momento de decisión sobre una medida cautelar. Por ejemplo, se suele hablar de "antecedentes", "presunciones", "bases para una presunción" En términos sencillos, pareciera que la prueba en el sistema procesal es aquella que tiene lugar en la audiencia de juicio oral pues el legislador usa ese vocablo (prueba o medios de prueba) cuando se diseñan las condiciones para la decisión del juicio. Parte de la doctrina ha visto en este punto una alocación de la prueba en la audiencia de juicio oral. Luego, sólo sería prueba lo que se produce en la audiencia de juicio. Los antecedentes recabados con anterioridad no merecen ser llamados, en este sentido, prueba. ${ }^{18}$

Sin embargo, hay actividad probatoria exactamente donde se trate de corroborar la veracidad de un enunciado que sirva de base para una decisión. Hay por tanto prueba en contextos como el diagnóstico médico, las decisiones de diseño y ejecución de políticas públicas, el diseño, la arquitectura, la ingeniería, y así, en cualquier campo racional de adopción de decisiones.

Esto ocurre en el proceso penal en diversos casos, y por cierto, en la decisión de adopción de una determinada medida cautelar en la medida en que pretendamos que esa decisión sea racional y permita verificar sus razones. Renunciar a la actividad probatoria en este ámbito para situarlo tan sólo en el contexto de juicio oral supone convertir la decisión cautelar en una decisión arbitraria. ${ }^{19}$

\footnotetext{
${ }^{17}$ Como ya ha sido apuntado desde Bentham, la prueba jurídica nos permite enfrentar el sentido del uso del derecho. Véase en particular a lo tocante al razonamiento con hechos contrarios a las leyes de la naturaleza (dentro de los cuales pueden inscribirse los hechos del futuro) BENTHAM, Jeremías, "Tratado de las pruebas judiciales", Paris: Bosange Frères, 1825, p. 242.

${ }_{18}$ Así por ejemplo pareciera quedar establecido de la lectura del artículo 296 del Código Procesal Penal: "Artículo 296. Oportunidad para la recepción de la prueba. La prueba que hubiere de servir de base a la sentencia deberá rendirse durante la audiencia del juicio oral, salvas las excepciones expresamente previstas en la ley. En estos últimos casos, la prueba deberá ser incorporada en la forma establecida en el párrafo $9^{\circ}$ de este título". Sin embargo, debemos considerar que esta disposición regula una clase de actividad probatoria: la relativa al juicio oral y a la sentencia. Ello no descarta, sin más, la posibilidad de entender que el razonamiento probatorio se encuentra disponible en otros escenarios procesales en los que deba adoptarse alguna decisión basada en hechos. Una visión contrapuesta es la defendida por Julián López. Véase HORVITZ, María y LÓPEZ, Julián, "Derecho Procesal Penal chileno", cit. Nota 5, pp. 68 - 69.

${ }^{19}$ Esto provocaría una primera contradicción para la institución denominada "prueba ilícita" en tanto prueba que manifiesta defectos de legitimidad. Si existe prueba que tenga un defecto tal que permita denominarla
} 
Particularmente en el caso de la prisión preventiva aparece un modo en que estos hechos pueden ser considerados como razón justificatoria de la aplicación de esa medida. Es decir, la decisión de esa medida descansa, como toda aplicación del derecho en dos clases de premisas: una premisa de derecho o normativa y una premisa de hecho o fáctica. A esta segunda premisa nos dirigimos cuando nos enfrentamos a problemas de pruebas y a la necesidad de determinar las reglas según las cuales deba afirmarse que determinada aserción sobre los hechos del caso (cautelar) deban entenderse concurrentes.

Por ejemplo, los hechos que configuran el delito de cuya investigación se trate pueden ser corroborados de modo tal que cuenten como un dato provisional para aplicar la medida bajo la lógica de la proporcionalidad. En general estos datos suelen ser llamados "antecedentes", ¿Qué diferencia hay entre un antecedente que justifique a la prisión preventiva y evidencia acerca de la plausibilidad de los hechos que justifican a la prisión preventiva?

Creo que es más o menos evidente que dado que nos encontramos ante una decisión racional de relevancia en el contexto del procedimiento sus premisas fácticas deben someterse a algún grado, aunque sea mínimo, de corroboración.

\section{5. ¿Sobre qué debe decidirse? Enunciados sobre hechos y predicciones.}

Probablemente el primer análisis que realice un juez frente al relato de un hecho sea el que se dirige a determinar si existe plausibilidad en el relato que se le presenta. Todo conocimiento sobre hechos está mediado por la necesidad de evaluar los relatos a través de los cuales el conocimiento se comparte. En esta medida la actividad judicial es, en gran medida, un tipo de actividad vinculada a esa clase de evaluaciones. ${ }^{20}$

Es difícil imaginar que un juez pudiera aceptar como una explicación razonable acerca de los antecedentes que permiten afirmar la necesidad de aplicación de una medida cautelar una explicación que resulte abiertamente ilógica o insensata.

Si se señala que un sujeto que está sometido por primera vez al control penal y que manifiesta una actitud colaborativa respecto de la investigación necesite ser conducido a prisión preventiva por constituir un peligro para la seguridad de la sociedad debido a su afición a coleccionar, por ejemplo, estampillas, resulta claramente inadecuado que el juez pueda afirmar que concurre ese supuesto para aplicar la prisión preventiva.

\footnotetext{
ilícita y por tanto excluirla de la audiencia de juicio oral, en realidad no es ni habrá sido "prueba". En esta medida la expresión "prueba ilícita" comportaría una contradicción en los términos. No es este el lugar para hacerlo, pero las exclusiones probatorias, tradicionalmente entendidas como reglas "contra-epistémicas" contienen muchos puntos de interés para una tesis comprometida con una explicación del proceso vinculada a la averiguación de la verdad.

${ }^{20}$ En esto consiste en gran medida la confianza en los modelos empiristas, véase GASCON, Marina, "Los hechos en el derecho. Bases argumentales de la prueba", Barcelona: Marcial Pons, 2010, p. 16.
} 
VALENZUELA, Jonatan. "Hacia un estándar de prueba cautelar en materia penal: algunos apuntes para el caso de la prisión preventiva".

Posiblemente el mejor modo de enfrentamiento que el juez tenga disponible para evaluar los relatos presentados por las partes respecto de las medidas cautelares sea la denominada "plausibilidad" derivada del esquema de una teoría general sobre estructura de la prueba. ${ }^{21}$ Este primer examen supone la definición de una relación entre los hechos que son corroborados en la audiencia con aquellos que buscamos evitar a través de la medida cautelar (v.gr. la fuga del imputado). En alguna medida las evidencias sobre hechos deben permitir la afirmación de verdad de un enunciado considerando la probabilidad de ocurrencia de un determinado hecho en el futuro que permita justificar la medida cautelar.

Ese ejercicio es el propio de la aplicación de las denominadas inferencias probatorias epistemológicas: la observación de cierta regularidad en el acaecimiento de ciertos hechos permite sostener que nos encontramos ante un relato plausible o no. Si el imputado enfrenta una investigación robusta respecto de un delito que tiene una alta pena, es posible entender que su deseo de fugarse es mayor que el del que enfrenta una investigación exigua respecto de un delito que sólo tiene pena de multa. ${ }^{22}$

Así, la idea de máximas de experiencia permite dotar de un campo de acción al juez para determinar si cabe esperar razonablemente el acaecimiento de un hecho en el futuro que justifique la imposición de la prisión preventiva. Ciertamente, esto no es necesario respecto de todos los puntos que pueden exigirse para decidir sobre una medida de este tipo, pero claramente es capital cuando se trata de criterios que miran al futuro. ${ }^{23}$

Si volvemos al ejemplo del coleccionista de estampillas tenemos que el hecho acreditado de poseer una larga y formidable colección de estampillas no permite sostener que se sigue desde ahí cierto peligro. Por otro lado, la compra de pasajes aéreos por parte del imputado puede justificar (junto a otras circunstancias) la necesidad de imponer una medida cautelar por peligro de fuga. En este último caso pues la predicción se basa en la regularidad empírica por la cual quienes han adquirido pasajes aéreos suelen viajar.

Ese razonamiento es propio de la aplicación de reglas de epistemología general, en las que el relato presentado debe ser sometido a la lógica, generalizaciones empíricas, y en un terreno siempre dudoso, a información científica.

\footnotetext{
${ }^{21}$ En este sentido FERRER, Jordi, "Prueba es libertad pero no tanto: una teoría de la prueba cuasi benthamiana" en VAZQUEZ, Carmen, "Estándares de prueba y prueba científica”, Barcelona: Marcial Pons, 2013, p. 27.

${ }^{22}$ Las inferencias probatorias pueden ser conforme a la visión defendida por Daniel González de tres clases: epistemológicas, normativas e interpretativas. Las primeras toman la forma de inferencias que se amparan en las observaciones de regularidades empíricas comúnmente aceptadas (máximas de experiencia). Las segundas corresponden a definiciones del derecho sobre concurrencia de determinados enunciados probatorios (como ocurre con las presunciones). Las terceras se relacionan con el uso de conceptos que puede utilizar el juez para definir, clasificar u otras operaciones respecto de los enunciados probatorios. Sobre el particular, véase GONZALEZ, Daniel, "Presunción de inocencia, verdad y objetividad" en GARCIA, Juan y BONORINO, Pablo (Coords.) "Prueba y razonamiento probatorio en derecho. Debates sobre abducción", Madrid: Comares, 2014, pp. 83-117, pp. 85 - 90.

${ }^{23} \mathrm{Si}$ bien no puede afirmarse absolutamente que toda inferencia probatoria epistémica sea una máxima de experiencia, me parece que toda máxima de experiencia es una clase de inferencia probatoria epistémica. Este es el sentido asumido en este trabajo.
} 
Este no es el único tipo de inferencias probatorias que parece tener lugar. También pueden concurrir determinadas inferencias probatorias normativas. ${ }^{24}$

Es el caso de la presunción de inocencia como regla de clausura frente a la incertidumbre fáctica en las medidas cautelares penales. Se ha sostenido, erróneamente a mi juicio, que la presunción de inocencia tiene lugar en tanto expresión de una "anticipación punitiva". Es decir, se afirma que no es posible compatibilizar el respeto por la presunción de inocencia y a la vez hacer uso de la prisión preventiva. En otro trabajo me he ocupado de este punto mostrando que la lógica de despliegue de la pena es la que permite desatar el uso de la presunción de inocencia y que las medidas cautelares, como la prisión preventiva no tiene la aptitud para cuestionar el estatuto de inocente del sometido a prisión preventiva. Quien padece una prisión preventiva debe ser considerado normativamente inocente y por tanto la presunción de inocencia no constituye en realidad una prohibición para la prisión preventiva. $^{25}$

Puede pensarse que la misma presunción opera como regla probatoria, más precisamente como una inferencia probatoria normativa que permite sostener que los casos de incertidumbre (determinados por las inferencias normativas epistemológicas) deben ser considerados a favor del imputado. La fenomenología del encierro muestra que el error al conceder una medida cautelar de prisión preventiva que no debía concederse es de un alto peso y por tanto debe entenderse así su aplicación restrictiva. ${ }^{26}$

En este sentido, la presunción de inocencia debe ser entendida como una regla que forma parte del modelo de razonamiento probatorio del juez. Como toda presunción es una regla que afirma que dada la prueba del evento $p$ debe presumirse como verdadero el evento desconocido $q$, el que puede ser entendido como cualquier evento compatible con la inocencia del acusado y en particular con hechos que puedan derrotar a aquellos que puedan justificar a la cautelar. $^{27}$

Sin embargo, ¿cómo justificar la suficiencia de cierta evidencia para prever el acaecimiento de hechos?

Creo que debemos distinguir entre predicciones y meras especulaciones. Yo puedo especular con la posibilidad, al parecer difícil, de que la selección de fútbol de Chile vaya a ganar el campeonato mundial de fútbol de 2022, y en esta medida puedo tomar decisiones

\footnotetext{
${ }^{24}$ Véase sobre las inferencias probatorias normativas el mismo texto de GONZALEZ, Daniel, op. cit. nota 22.

${ }^{25}$ Véase VALENZUELA, Jonatan, Presumir responsabilidad: sobre una relación entre la prisión preventiva y la presunción de inocencia en el derecho procesal chileno", en Revista de Estudios de la Justicia, núm. 14, (2011) pp. $53-70$.

${ }^{26}$ A esto se refiere la idea de excepcionalidad de las medidas cautelares personales, véase HORVITZ, María y LÓPEZ, Julián, "Derecho procesal penal chileno"....cit. Nota 5, p. 352.

${ }^{27}$ Sobre la noción de presunción resulta ineludible ULLMAN MARGALIT, Edna, “On Presumption” en The Journal of Philosophy, Vol. 80 №3, 1983, pp. 143 - 163. Para una tipología de las presunciones véase GAMA, Raymundo, "Concepciones y tipología de las presunciones en el derecho continental”, en Revista de Estudios de la Justicia (2013) pp. 65 - 89.
} 
VALENZUELA, Jonatan. "Hacia un estándar de prueba cautelar en materia penal: algunos apuntes para el caso de la prisión preventiva".

variadas amparadas en esa "aspiración", especular es, en alguna medida, aspirar y tentar a la suerte.

Sin embargo, la predicción supone necesariamente a la evidencia. Ante la misma pregunta, relativa a las posibilidades de la selección chilena en el campeonato mundial de fútbol de 2022 puede responderse de manera escéptica citando los antecedentes que la selección de fútbol de Chile parece exponer como potencial candidato a ganar el título mundial. De hecho, una predicción puede volverse contextualmente precisa en la medida que se requiere determinar, por ejemplo, las posibilidades de que la selección chilena elimine a la selección de Brasil en segunda fase.

Podemos especular con una victoria inédita, pero lo más probable es que podamos predecir una derrota conforme a la regularidad de los sucesos vinculado a los partidos entre Chile y Brasil.

En las medidas cautelares, dado el contexto probatorio que parece quedar definido, debemos orientar la actividad de valoración de antecedentes, en una clase de predicción y no de especulación.

La necesidad de tomar decisiones basadas en evidencia supone desentrañar el criterio con el cual el juzgador debe enfrentar la verificación de las premisas fácticas del caso. En esta medida, que concurren diversas inferencias probatorias en la decisión de imponer una prisión preventiva. La pena asignada al crimen, o las condenas anteriores, o la sujeción a una medida cautelar previa, permiten ser situadas en el terreno de las inferencias probatorias normativas.

Es el legislador el que ha realizado ya sea una búsqueda de consagración de una regularidad empírica o de protección de ciertos valores, y queda por tanto en el nivel judicial, la necesidad de aplicar una regla.

Sin embargo, otras circunstancias, como por ejemplo la pretendida presunción de que el imputado realizará atentados contra la víctima o contra su familia o bienes como base para una presunción de peligro, en realidad denota una clase de inferencia probatoria epistemológica. Estas inferencias requieren la identificación de una regla de estándar probatorio particular para el caso de la prisión preventiva. ${ }^{28}$

\section{Hacia una propuesta concreta de estándar de prueba para la prisión preventiva.}

Los aspectos del razonamiento probatorio cautelar son múltiples. Lo que debemos determinar, en un trabajo exploratorio como este, es la clase de estándar de prueba que puede ser aplicado en este contexto. Esta definición es en realidad básica de cara a la afirmación del razonamiento basado en evidencias, dado que la regla de estándar de prueba tiene una función heurística que tiende a definir toda la actividad probatoria. La definición

\footnotetext{
${ }^{28}$ La expresión presunción en ese escenario está referida en realidad a un tipo de razonamiento probatorio y no presuntivo. Dado que la observación de la regularidad entre un evento Q que debe entenderse concurrente y un evento P descansa en el juez, esta sería con mucho, una falsa presunciones. Véase al respecto GAMA, Raymundo, op. cit. nota 26.
} 
Polit. crim. Vol. 13, No 26 (Diciembre 2018) Art. 5, pp. 836-857.

[http://www.politicacriminal.cl/Vol_13/n_26/Vol13N26A5.pdf]

de la regla de estándar de prueba es la piedra angular de toda decisión cuya justificación racional se pretenda.

El principal riesgo de no tematizar la necesidad de contar con una regla de estándar probatorio es llevar a que las decisiones judiciales que se adopten al respecto resulten mediadas por el azar, por consideraciones poco igualitaristas y en definitiva den pie a la arbitrariedad.

La primera consideración derivada de la necesidad de coherencia del sistema probatorio general es que no puede tratarse del estándar de juicio. Si en un sistema procesal se decide por una formulación (las más de las veces vaga) de una exigente duda razonable como estándar de prueba orientada a hechos del pasado, pareciera que el estándar de prueba cautelar debe ser diferente. $^{29}$

La diferencia radica en primer término en la arquitectura de la decisión probatoria en materia cautelar que debe incluir la evaluación de enunciados respecto de hechos que se espera acaezcan en el futuro. Esto es propio del razonamiento cautelar y no es común en el uso de estándares de prueba de juicio. En este escenario, la diferencia del razonamiento no radica en la mayor o menor exigencia del mismo estándar. Por ejemplo, pareciera que la potencial imposición de una cautelar de prisión preventiva describe un error judicial potencial empíricamente muy similar al de la pena. Es decir, parece que desde el punto de vista de la facticidad de la decisión, es difícil ignorar el hecho de que en uno y otro caso se envía a una persona al encierro. En este sentido, es posible que la exigencia probatoria cautelar propia de la prisión preventiva sea alta en comparación a las otras medidas cautelares. Esto porque el error potencial en la imposición de una citación parece de menor entidad fenomenológica que el de la prisión.

La razón principal es la clase de error que supone una y otra decisión. A diferencia de la sentencia, la decisión sobre aplicación de una medida cautelar implica, como hemos apuntado, su provisionalidad y su instrumentalidad. Esto muestra que se trata de decisiones provisionales en las que se cuenta con una determinada información (muchas veces baja) diferente respecto de la que deberíamos entender que se usará en el momento del juicio oral.

Por ello, el error que se encuentra en juego en las medidas cautelares tiene caracteres diferentes al error de condena o absolución propio de la sentencia. La concesión de una cautelar en un caso donde no cabía dicha medida o su denegación en un caso en el que debía ser acogida comportan un daño a bienes de intensidad similar al de la pena en algunos casos, pero que por definición resulta provisional. Es evidente que se requiere actividad

\footnotetext{
${ }^{29}$ Ciertamente uno debe estar dispuesto a discutir este lugar común acerca de la duda razonable. Que sea un estándar exigente depende directamente de la clase de exigencia que sea capaz de expresar ese estándar de prueba y esto depende de la manera en que se dote de contenido a la regla de estándar. Por ahora creo que lo que debe resaltarse es el compromiso necesario que aparece con el desplazamiento de hipótesis explicativas de los datos disponibles que sean compatibles con la inocencia del acusado. Este punto es el que detonaría una clase de exigencia elevada del estándar de juicio frente al cautelar.
} 
VALENZUELA, Jonatan. "Hacia un estándar de prueba cautelar en materia penal: algunos apuntes para el caso de la prisión preventiva".

probatoria tendiente a evitar el error que puede ser distribuido a través de la regla de estándar.

Por otro lado, dada la estructura de las medidas cautelares debemos entender que son medidas en las que no aparece en juego la presunción de inocencia en su vertiente de regla de juicio. Es decir, la calidad de "inocente" del imputado no se encuentra en juego debido a que la decisión que tiene la aptitud de generar el uso de una regla de estándar de prueba de juicio es la decisión de absolución o condena.

En este escenario deberíamos preguntarnos por una propuesta de estándar de prueba penal para proponer un estándar de prueba cautelar penal.

Ferrer sostiene que el estándar de prueba penal debe ser entendido conforme a los siguientes pasos:

“1) La hipótesis debe ser capaz de explicar los datos disponibles, integrándolos de forma coherente, y las predicciones de nuevos datos que la hipótesis permita formular deben haber resultado confirmadas.

2) Deben haberse refutado todas las demás hipótesis plausibles explicativas de los mismos datos que sean compatibles con la inocencia del acusado, excluidas las meras hipótesis ad hoc., 30

La satisfacción de este estándar de prueba supone que la duda razonable queda integrada por la necesaria explicación coherente de los datos probatorios, es decir, por la capacidad que tienen las evidencias de apoyar una determinada hipótesis de hecho. Además, esos datos deben ser capaces de justificar la formulación de predicciones, entendidas como descubrimiento de datos desconocidos basados en los conocidos, cuya confirmación refuerza la conclusión probatoria.

Además este estándar de prueba supone el desplazamiento de las hipótesis compatibles con la inocencia del acusado, es decir, supone que la incertidumbre sobre el escenario probatorio puede beneficiar al inocente (dando cumplimiento a la presunción de inocencia como regla de juicio).

Como se ve esta exigencia expresa la necesidad de "peso" de la hipótesis que se ha mostrado comparativamente mejor en términos de explicación de los datos disponibles.

Con las consideraciones antedichas, y en comparación a la exigencia del estándar de prueba presentado, un estándar de prueba cautelar penal debería sostener:

1) La hipótesis de hecho debe ser capaz de explicar los datos disponibles, integrándolos de forma coherente;

2) Las predicciones de nuevos datos que la hipótesis permita formular deben haber resultado confirmadas;

\footnotetext{
${ }^{30}$ FERRER, Prueba es libertad... cit. Nota 7.
} 
Polit. crim. Vol. 13, No 26 (Diciembre 2018) Art. 5, pp. 836-857.

[http://www.politicacriminal.cl/Vol_13/n_26/Vol13N26A5.pdf]

3) Deben formularse predicciones prospectivas basadas en las evidencias que permitan que se acepte como la hipótesis de mayor peso aquella compatible con la ocurrencia de un hecho en el futuro.

En el punto 1 propuesto, tenemos que dado el enfrentamiento a un escenario de actividad probatoria, los enunciados sobre hechos que den lugar a la cautela deben apoyarse en evidencias producidas por el persecutor. Esos datos deben ser integrados de modo coherente con una versión preliminar de los hechos acaecidos en el pasado permitiendo tenerlos por verdaderos provisionalmente. Lo que se requiere de las evidencias en este punto no es diferente de lo requerido ante la decisión de juicio, y ello es razonable pues es la mínima exigencia de racionalidad de la decisión.

En los puntos 2 y 3 se contemplan dos clases de predicciones, a las que llamaré "predicciones cautelares de primer orden" y "predicciones cautelares de segundo orden".

En el punto 2, es decir sobre las predicciones cautelares de primer orden, deben realizarse predicciones de nuevos datos que resulten confirmadas (por ejemplo si se investiga por un delito cometido usando un arma de fuego, puede confirmarse la hipótesis relativa a la presencia de pólvora en las manos del imputado). Estas predicciones permiten un avance desde los datos conocidos hacia datos desconocidos, que pueden mejorar la hipótesis explicativa de los hechos.

En este paso el juicio sobre las evidencias sigue dirigido al pasado reforzando la tesis cuya coherencia ha sido integrada en el punto 1. Lo particular de este grupo de predicciones es que se avocan al pasado, es decir, constituyen un tipo de indagación reconstructiva del supuesto de hecho que da lugar a la prueba de la premisa de hecho de aplicación de la medida cautelar.

En cambio, cuando se trata de predicciones cautelares de segundo orden (punto 3) se propone realizar un ejercicio predictivo abiertamente prospectivo, que permita tener por verdadero un enunciado pues resulta razonable esperar el acaecimiento de un hecho que justifique la imposición de la medida. Ello, con independencia del efectivo acaecimiento de ese hecho en el futuro. Es claro que ese razonamiento se hace con referencia a hechos, y por tanto, con información del pasado. En alguna medida supone la afirmación de cierta capacidad de proyección de una inferencia probatoria.

Dicho en otros términos, el razonamiento probatorio cautelar supone anticipar una clase de acaecimientos y la aceptación de la actividad cautelar depende de la racionalidad de la afirmación de la premisa sobre necesidad de cautela que queda enmarcada en las predicciones cautelares de segundo orden.

En este sentido, -y como he apuntado anteriormente- la herramienta que surge para el juzgador es la formulación de máximas de experiencia en tanto inferencias probatorias epistémicas. Como se sabe, las máximas de experiencia forman parte de los modelos de valoración probatoria permitiendo la afirmación de acaecimiento de un hecho dada la concurrencia de información regular en el pasado. De esto modo, resulta importante dar 
VALENZUELA, Jonatan. "Hacia un estándar de prueba cautelar en materia penal: algunos apuntes para el caso de la prisión preventiva".

espacio a razonamientos que permitan la aceptación del acaecimiento de estos hechos asumida la capacidad que tiene la información acerca del pasado para permitir predecir el acaecimiento de un hecho en el futuro. La fuerza de esta inferencia dependerá de la fuerza que tenga la máxima dado su apoyo en la observación empírica. ${ }^{31}$

Aquí aparece el potencial impacto del principio de proporcionalidad de las medidas cautelares. Si las predicciones de segundo tipo permiten considerar como plausible que el imputado se fugue, entonces parece que se habilita razonablemente al juez para imponer una prisión preventiva.

El razonamiento predictivo de este tercer paso debe constituir un avance también desde datos conocidos hacia desconocidos. Pero, a diferencia de las predicciones de primer orden, estas se orientan al futuro sosteniendo la alta probabilidad de acaecimiento de un hecho en el futuro. Este es un juicio propio del escenario de las regularidades empíricas, esto es, supone la asociación de dos eventos en un marco de determinación de probabilidad de ocurrencia de un evento aceptada la observación del mundo a través de los hechos que han tenido lugar.

Además ese razonamiento no es totalmente comparativo sino que tiende a la definición del "peso". Esto quiere decir, que debe entenderse que la hipótesis que se considera como plausible en este tercer paso debe considerarse con un peso suficiente de cara a la existencia de evidencias que le brinden apoyo. Es a partir de evidencias que podemos realizar predicciones.

Lo interesante es considerar que estas predicciones deben dejarse explicar a partir de los pasos 1 y 2 anteriormente propuestos. Es decir, debe tratarse de predicciones que queden explicadas a partir de la coherencia exigida en el punto 1 y de la confirmación de las predicciones de primer orden.

Lo distintivo de este estándar de prueba frente al estándar propio del juicio oral y la sentencia es que debe prescindir del desplazamiento de las hipótesis alternativas de cara a la aplicación de la regla de juicio derivada de la presunción de inocencia.

Este es un caso donde esa regla no recibe aplicación y el juez puede entender que hay suficiencia de la evidencia de la medida cautelar aún en casos en que pervivan hipótesis de hecho compatibles con la inocencia del acusado.

\section{Proyecciones en la regulación sobre prisión preventiva en Chile.}

Es necesario mostrar como las anteriores ideas pueden proyectarse operativamente en el contexto de la regulación chilena sobre prisión preventiva.

\footnotetext{
${ }^{31}$ Sobre las máximas de experiencia véase NAVARRO, René, "Bases para una sana crítica. Lógica, interpretación, argumentación, máximas de la experiencia, conocimiento científico”, Santiago: RIL Editores, 2014, p. 123.
} 
Polit. crim. Vol. 13, No 26 (Diciembre 2018) Art. 5, pp. 836-857.

[http://www.politicacriminal.cl/Vol_13/n_26/Vol13N26A5.pdf]

Las medidas cautelares en materia penal deben ser enfrentadas al razonamiento probatorio en tanto modelo de evaluación de la verosimilitud de los elementos de juicio disponible para enfrentar a los hechos que dan lugar a la aplicación de las reglas jurídicas que permiten encerrar cautelarmente a alguien.

Probablemente el escenario normativo más general donde podamos afirmar esta necesidad esté en lo dispuesto en el artículo 122 del Código Procesal Penal. Como se sabe, esta regla establece que las medidas cautelares personales en materia penal sólo pueden imponerse cuando fueren absolutamente indispensables para asegurar los fines del procedimiento y sólo durarán mientras subsistiere la necesidad de su aplicación. ${ }^{32}$

Lo más relevante es que siempre serán decretadas por medio de resolución judicial fundada. En la perspectiva aquí defendida, la fundamentación de la resolución pareciera tener que abarcar, ciertamente, una determinada cuestión de hecho. No parece posible entender la existencia de una resolución fundada sin que medie un pronunciamiento del juez respecto de los enunciados acerca de los hechos del asunto que debe resolver. Y sabemos, que parte de las aserciones que deben formularse en la resolución respecto de los hechos debe referirse sobre algo que en realidad no está referido a hechos, pues se trata de eventos futuros.

Dado este escenario, resulta evidente que las reglas sobre procedencia de las medidas cautelares deben someterse a reglas sobre prueba. ¿Qué es lo común a todas ellas? A mi juicio, el enfrentamiento de una regla de estándar de prueba como la propuesta.

Ciertamente el grupo más destacado de reglas que merecen ser enfrentadas en esta línea se encuentran en el artículo 140 del CPP.

En este grupo, y asumiendo la perspectiva del razonamiento probatorio cautelar que ha sido hasta aquí expuesta, creo que puede distinguirse entre hipótesis que son claramente retrospectivas, es decir, suponen la justificación de una decisión judicial que mira al establecimiento de hechos cuyo acaecimiento se verifica en el pasado, y por otra parte existen reglas que suponen que el juez deba justificar decisiones probatorias con enunciados que miran al acaecimiento de hechos en el futuro.

Lo común a estas dos clases de enunciados probatorios es que conforme al inciso primero del artículo 140 CPP el solicitante debe acreditar un enunciado fáctico. La acreditación es, a mi juicio, una apelación a la prueba de estos enunciados, es decir, un llamado a establecer condiciones para afirmar la verdad o falsedad de los enunciados acerca de los hechos que pueden justificar la decisión probatoria de la medida cautelar personal de prisión preventiva.

\footnotetext{
${ }^{32}$ El artículo 122 dispone: "Finalidad y alcance. Las medidas cautelares personales sólo serán impuestas cuando fueren absolutamente indispensables para asegurar la realización de los fines del procedimiento y sólo durarán mientras subsistiere la necesidad de su aplicación.

Estas medidas serán siempre decretadas por medio de resolución judicial fundada.”
} 
VALENZUELA, Jonatan. "Hacia un estándar de prueba cautelar en materia penal: algunos apuntes para el caso de la prisión preventiva".

En el primer grupo o clase encontramos los supuestos de la letra a) y b) del mencionado artículo 140 CPP. En estos casos, el juez debe adoptar una decisión claramente retrospectiva. En el caso de la letra a) del artículo 140 del CPP, debe poder afirmar la existencia del delito. En este sentido la exigencia probatoria se encuentra fuertemente emparentada con la lógica de la sentencia, pues debe poder considerarse como verdadero el enunciado sobre la ocurrencia del delito. Es evidente que no se trata de la justificación de una condena, por lo que el juez puede considerar plausible la concesión de la prisión preventiva con la sola prueba de enunciados sobre existencia del delito sin realizar todavía un juicio de atribución afinado sobre responsabilidad penal del imputado.

En este primer grupo encontramos también a la letra "b" del mismo artículo 140. En ella se expresa que se tendrá por probado, en términos cautelares, la participación a través de presunciones fundadas. Presumir fundadamente debería oponerse a presumir de manera infundada. Parece ser que, si se sigue un esquema clásico de presunciones, de lo que se trata es de permitir dar por concurrente la conexión entre el imputado y los hechos de la letra a) por medio de una presunción judicial, es decir, que el juez pueda entender al hecho presumido (la participación) como aceptable dada la prueba del hecho base, esto es, indicios sobre la relación entre el delito de la letra a y la participación. Es claro que este ejercicio de razonamiento no podría por sí dar lugar a una condena, de otro modo el juez podría adelantar completamente el juicio. Por otro lado, no contamos con una presunción del tipo de la presunción de inocencia en el marco de una decisión que no puede declarar al sujeto como culpable. ${ }^{33}$

Esta inferencia probatoria tiene la particularidad de permitir al juez actuar con la información disponible asumiendo un escenario de incertidumbre sobre la participación a efectos de justificar a la prisión preventiva. Por lo tanto, en estos casos las presunciones deben ser entendidas como apelaciones a la actividad probatoria que debe tener lugar en el escenario de adjudicación de la prisión preventiva. Hasta aquí la decisión probatoria cautelar de la prisión preventiva puede enfrentarse con la verificación de la regla de estándar de prueba propuesta en particular en los puntos 1 y 2 anteriormente señalados, esto es se trata de definir si la hipótesis de hecho debe ser capaz de explicar los datos disponibles, integrándolos de forma coherente y si las predicciones de nuevos datos y sus correlativas hipótesis de hecho deben haber resultado confirmadas.

En el segundo grupo, encontramos los criterios agrupados en la letra c) del artículo 140.

En esta clase de criterios encontramos que debe atenderse a la existencia de "antecedentes calificados que permitieren al tribunal considerar que la prisión preventiva es indispensable para el éxito de diligencias precisas y determinadas de la investigación, o que la libertad del imputado es peligrosa para la seguridad de la sociedad o del ofendido, o que existe peligro de que el imputado se dé a la fuga". Como puede apreciarse se trata de supuestos de hecho que se producirán, son "hechos futuros" o más precisamente enunciados sobre situaciones que aún no constituyen aquello que llamamos claramente "hechos" en sentido probatorio.

\footnotetext{
33 Véase sobre este particular el trabajo de GAMA, Raymundo, "Concepciones y tipología de las presunciones..." cit. nota 20 .
} 
Como se sabe, una propiedad evidente de estos supuestos de hechos es que al situarse en el futuro, a diferencia de los del primer grupo, no pueden darse por probados en el esquema de razonamiento probatorio tradicional que busca la reconstrucción de los hechos del pasado para definir la verdad o falsedad de los enunciados sobre esos mismos hechos. Por ello, resulta claro que nos encontramos en un terreno en el que la posibilidad de error en la decisión aparece con intensidad habida consideración de que al tratarse de hechos futuros estos pueden ocurrir o no.

En el inciso segundo de la letra c) del artículo 140 CPP nos encontramos con que existe una regla abierta para realizar inferencias probatorias sobre la base de toda la diversidad de datos que puedan aparecer. Se trata de advertir una "sospecha grave y fundada de que el imputado pudiere obstaculizar la investigación mediante la destrucción, modificación, ocultación o falsificación de elementos de prueba; o cuando pudiere inducir a coimputados, testigos, peritos o terceros para que informen falsamente o se comporten de manera desleal o reticente".

¿Cuál es la base de ese razonamiento judicial? Pareciera que hasta aquí el legislador pone el juez en situación de especular respecto del hecho de la producción futura de un hecho que se deje describir como una fuga, como manipulación o destrucción de pruebas.

En el caso de los supuestos de los incisos tercero y cuarto de la letra c) del mismo artículo tenemos que la situación varía levemente. Por una parte, para afirmar la conducencia de la afirmación de peligrosidad del imputado para la seguridad de la sociedad el juez debe justificar afirmaciones de hecho claramente determinadas: la gravedad de la pena asignada el delito, el número de delitos que se imputa y su carácter, la existencia de procesos pendientes y si se ha actuado en grupo o pandilla. En estos casos, de manera indirecta el juez debe decidir sobre la base de la corroboración de estos supuestos para poder afirmar la peligrosidad del imputado para la seguridad de la sociedad y por esa vía dar por concurrente (junto a otros supuestos que veremos enseguida) que el enunciado acerca de la peligrosidad del imputado resulta correcto.

En el caso del inciso cuarto de la letra c) del artículo 140 resulta claro que se trata de una regla de concreción del inciso tercero. En este caso el legislador prescribe que debe entenderse especialmente concurrente la hipótesis de peligro para la seguridad de la sociedad el hecho de que los delitos tengan asignada pena de crimen, cuando existiere condena anterior por un delito que tenga igual o mayor pena, cuando se encontrare sujeto a alguna medida cautelar personal, en libertad condicional o gozando de alguno de los beneficios alternativos a la ejecución de las penas privativas o restrictivas de libertad contemplados en la ley.

Aquí resulta también claro que el escenario que el juez enfrenta es más bien el de corroboración de los enunciados realizados por los intervinientes acerca de los supuestos de hechos que justifican, otra vez de manera indirecta, la concurrencia del peligro para la 
VALENZUELA, Jonatan. "Hacia un estándar de prueba cautelar en materia penal: algunos apuntes para el caso de la prisión preventiva".

seguridad de la sociedad. Lo mismo puede decirse del resto de las hipótesis presentadas por el legislador en el artículo 140 del CPP.

Es en este grupo donde creo debe tenerse en cuenta el criterio propuesto en el número 3 del estándar de prueba propuesto más arriba. Se trata de determinar de cara al futuro si es posible formular predicciones prospectivas basadas en las evidencias que permitan que se acepte como la hipótesis de mayor peso aquella compatible con la ocurrencia de un hecho en el futuro.

El camino para realizar esta determinación, ciertamente, está expuesta a un error derivado del hecho de afirmar la concurrencia de un hecho que no ha tenido lugar y que no es por lo tanto en sentido estricto hecho en sentido probatorio. Para ello me parece que lo razonable es que el juez dirija sus esfuerzos a la definición de una máxima de experiencia a través de la corroboración de la fuerza de los datos con que avala la conclusión que le permite afirmar como hipótesis probatoriamente aceptable la concurrencia de un enunciado sobre un hecho que ha de tener lugar en el futuro y que justifique la imposición de la prisión preventiva.

\section{Conclusiones}

El razonamiento cautelar en materia penal debe ser sujetado a los marcos generales del razonamiento probatorio a efectos de poder dotar a la decisión judicial de criterios para decidir fundadamente la imposición de una prisión preventiva.

Para ello debemos considerar que el principal rasgo que define a la decisión de imposición de una prisión preventiva en tanto medida cautelar es la necesidad de establecer como hecho probatorio el acaecimiento de un evento futuro. Como sabemos, esto requiere formular una predicción.

Esas predicciones requieren aceptar enunciados referidos a ese acaecimiento futuro. Creo que deben realizarse basadas en las evidencias que permitan que se acepte como la hipótesis de mayor peso aquella compatible con la ocurrencia de un hecho en el futuro. El tipo de razonamiento típico para realizar esta operación tiene lugar a través de inferencias probatorias epistémicas, corrientemente, máximas de experiencia. La fuerza de las mismas depende de la fuerza que tenga la observación de los hechos que sirvan de aval a la afirmación de la máxima. Con una regla de estándar de prueba como la que aquí se propone puede distinguirse con claridad entre puntos probatorios que requieren de corroboración de cara al pasado (a través de la definición de capacidad de explicar los datos disponibles, integrándolos de forma coherente y de formulación de predicciones de nuevos datos y sus correlativas hipótesis de hecho deben haber resultado confirmadas) de aquellos que requieren corroboración de cara a la aceptación de un enunciado de cara al futuro.

En la legislación chilena, y particularmente en el contexto del artículo 140 CPP, tenemos que puede considerarse que las letras a) y b) del mismo tiende a situarse en la primera parte de la regla de estándar propuesta. En cambio los criterios de la letra c) de la misma disposición tienden a situarse en el contexto de verificación propio de las predicciones (que 
Polit. crim. Vol. 13, No 26 (Diciembre 2018) Art. 5, pp. 836-857.

[http://www.politicacriminal.cl/Vol_13/n_26/Vol13N26A5.pdf]

he denominado cautelares de segundo orden) y que miran al establecimiento de predicciones prospectivas basadas en las evidencias que permitan que se acepte como la hipótesis de mayor peso aquella compatible con la ocurrencia de un hecho en el futuro y con ello justifiquen la imposición de la prisión preventiva. 
VALENZUELA, Jonatan. "Hacia un estándar de prueba cautelar en materia penal: algunos apuntes para el caso de la prisión preventiva".

\section{Bibliografía}

ALVARADO, Adolfo, Cautela Procesal, Rosario: Juris, 2008.

ARAZI, Roland, Medidas Cautelares, Buenos Aires: Astrea, 2014.

BELTRÁN, Ramón, "Estándares de prueba y su aplicación sobre el elemento material de la prisión preventiva en Chile", Política Criminal, Vol. 7, No 14 (2012) pp. 454 - 479.

BENTHAM, Jeremías, Tratado de las pruebas judiciales, Paris: Bosange Frères, 1825.

DEI VECCHI, Diego, “Acerca de la justificación de la prisión preventiva y algunas críticas frecuentes”, Revista de Derecho (Valdivia), Vol. XXVI, N², 2013, pp. $189-217$.

FERRER, Jordi “Prueba y verdad en el derecho”, Barcelona: Marcial Pons, 2005.

FERRER, Jordi, "Prueba es libertad pero no tanto: una teoría de la prueba cuasi benthamiana", en VÁZQUEZ, Carmen, "Estándares de prueba y prueba científica", Barcelona: Marcial Pons, 2013.

GASCON, Marina, "Los hechos en el derecho. Bases argumentales de la prueba", Barcelona: Marcial Pons, 2010.

GAMA, Raymundo, (2013) "Concepciones y tipología de las presunciones en el derecho continental”, en Revista de Estudios de la Justicia, número 19, pp. 65 - 89.

GONZALEZ, Daniel "Presunción de inocencia, verdad y objetividad" en GARCIA, Juan y BONORINO, Pablo (Coords.) "Prueba y razonamiento probatorio en derecho. Debates sobre abducción”, Madrid: Comares, 2014, pp. 83-117.

HORVITZ, María y LÓPEZ, Julián “Derecho Procesal Penal Chileno, Tomo I”, Santiago: Editorial Jurídica de Chile, 2002.

HORVITZ, María y LÓPEZ, Julián “Derecho Procesal Penal Chileno, Tomo II”, Santiago: Editorial Jurídica de Chile, 2004.

KOSTENWEIN, Ezequial, "Prisión preventiva: entre los medios de comunicación y las autoridades políticas en Revista Direito e Práx., Rio de Janeiro, Vol. 08, N. 2, 2017, pp. $942-973$

LAUDAN, Larry "Verdad, error y proceso penal", traducción de Carmen Vázquez y Edgar Aguilera, Barcelona: Marcial Pons, 2013.

MARIN, Juan Carlos "Las medidas cautelares en el proceso civil chileno", Santiago de Chile: Editorial Jurídica, 2005. 
Polít. crim. Vol. 13, No 26 (Diciembre 2018) Art. 5, pp. 836-857.

[http://www.politicacriminal.cl/Vol_13/n_26/Vol13N26A5.pdf]

MATURANA, Cristian y MONTERO, Raúl, “Derecho Procesal Penal. Tomo I”, Santiago: Abeledo Perrot, 2010.

NAVARRO, Rene, "Bases para una sana crítica. Lógica, interpretación, argumentación, máximas de la experiencia, conocimiento científico”, Santiago: RIL Editores, 2014.

TARUFFO, Michele, “Simplemente la verdad”, Barcelona: Marcial Pons, 2010.

ULLMAN - MARGALIT, Edna (1983) “On Presumption”, en The Journal of Philosophy, Vol. 80, No 3, pp. $143-163$.

VALENZUELA, Jonatan (2011) "Presumir responsabilidad: sobre una relación entre la prisión preventiva y la presunción de inocencia en el derecho procesal chileno", en Revista de Estudios de la Justicia, núm. 14, pp. 53 - 70.

VALENZUELA, Jonatan, (2013) "Inocencia y razonamiento probatorio" en Revista de Estudios de la Justicia, número 18, pp. 13 - 23.

VALENZUELA, Jonatan, “Hechos, pena y proceso”, Santiago: Rubicón, 2017. 